\title{
Prevalence, distribution and antibiotic resistance of emergent Arcobacter spp. from clinically healthy cattle and goats
}

\begin{abstract}
Prevalence, distribution and antibiotic resistance of Arcobacter spp. were investigated in cattle, goats, floor and treated water samples in this study. The prevalence of Arcobacter in adult and young was recorded as $8 / 110(7.27 \%)$ and $4 / 83(4.81 \%)$, respectively, which showed insignificant difference $(\mathrm{P}=0.3503)$ in detection rates between adult and young cattle. A total of $33.33 \%$ of the floor samples and $11.11 \%$ of the treated water samples analysed were determined as positive for Arcobacter. Among the species isolated, over all, A. butzleri $(45 \%)$ was the most frequently detected species, followed by A. skirrowii (5\%). A. butzleri was isolated from adult cattle, floor and water samples at the rates of $75.0 \%, 33.4 \%$ and $50 \%$, respectively. Co-colonization of species was not uncommon, and $50 \%$ of the samples were carrying more than one Arcobacter species. Only $12.5 \%$ sample from cattle (adult) was detected positive for only A. skirrowii. All samples from young animals, floor and water contained mixed isolates. None of the samples from goat farm was found to be carrying Arcobacter species. On profiling of antimicrobial resistance patterns, it was found that only one A. butzleri isolate (3.7\%) was sensitive to all nine antibiotics tested. A. butzleri was found highly resistant to ampicillin (55.6\%), followed by cefotaxime $(33.4 \%)$ and ciprofloxacin (33.4\%). Overall, $20 \%$ of the isolates showed multidrug resistance (resistant $\geq 4$ antibiotics). Gentamicin and enrofloxacin can be used as drugs of choice for the treatment for Arcobacter infections
\end{abstract}

Keyword: Antibiogram; Arcobacter; Cattle; Goats; Prevalence. 